\title{
CONCENTRATION-DEPENDENT ABSORPTION AND EMISSION BEHAVIOUR OF A PYRIMIDONECARBOCYANINE DYE IN HEXAFLUOROISOPROPANOL
}

\author{
A. PENZKOFER, W. LEUPACHER, Bernd MEIER \\ Naturwissenschaftliche Fakultät II - Physik, Universität Regensburg, D-8400 Regensburg, FRG
}

B. RUNDE and K.H. DREXHAGE

Physikalisch-Chemisches Institut der Universität Siegen, D-5900 Siegen, FRG

Received 20 February 1987

\begin{abstract}
Absorption spectra, refractive index dispersions, fluorescence quantum distributions and fluorescence quantum efficiencies of a pyrimidonecarbocyanine dye in hexafluoroisopropanol are measured for various concentrations in the region between $10^{-5}$ and $0.3 \mathrm{~mol} / \mathrm{dm}^{3}$. The fluorescence quantum distributions and quantum efficiencies are separated into monomeric and dimeric (closely spaced pair) contributions. The fluorescence emission at high concentrations $C\left(>0.1 \mathrm{~mol} / \mathrm{dm}^{3}\right)$ is identified as closely spaced pair fluorescence. The absorption cross sections and the stimulated emission cross sections for the monomers and closely spaced pairs are resolved.
\end{abstract}

\section{Introduction}

The basic pyrimidonecarbocyanine dye 1,3, 1',3'-tetramethyl-2,2'-dioxopyrimido-6,6'-carbocyanine hydrogen sulfate (PYC, structure formula in fig. 1) has a very strong $S_{0}-S_{1}$ absorption band around $530 \mathrm{~nm}$ and shows very weak absorption at $350 \mathrm{~nm}$. These features make the dye a potential candidate for efficient conversion of intense picosecond Nd-glass laser pulses at $\lambda_{\mathrm{L}}=1.055 \mu \mathrm{m}$ to $\lambda_{3}=351.7 \mathrm{~nm}$ by phase-matched thirdharmonic generation [1-4].

The study of the dynamics of phase-matched third-harmonic generation requires information on the absorption, emission, and refractive index be-

Fig. 1. Absorption spectra of PYC in hexafluoroisopropanol. The curves belong to the concentrations $C=10^{-4}(1), 10^{-2}$ (2), 0.1 (3), 0.2 (4), and 0.3 (5) $\mathrm{mol} / \mathrm{dm}^{3}$. The structural formula of PYC is included. Curves 1 and 5 represent the monomer and closely spaced pair absorption cross sections, respectively. (The molar decadic absorption coefficient, $\epsilon_{\mathrm{A}}$, (dimension $\ell \mathrm{mol}^{-1} \mathrm{~cm}^{-1}$ ) is related to $\sigma_{\mathrm{A}}\left(\mathrm{cm}^{2}\right)$ by $\epsilon_{\mathrm{A}}=$ $\sigma_{\mathrm{A}} N_{\mathrm{A}} /[1000 \ln (10)], N_{\mathrm{A}}$ is Avogadro's constant.)

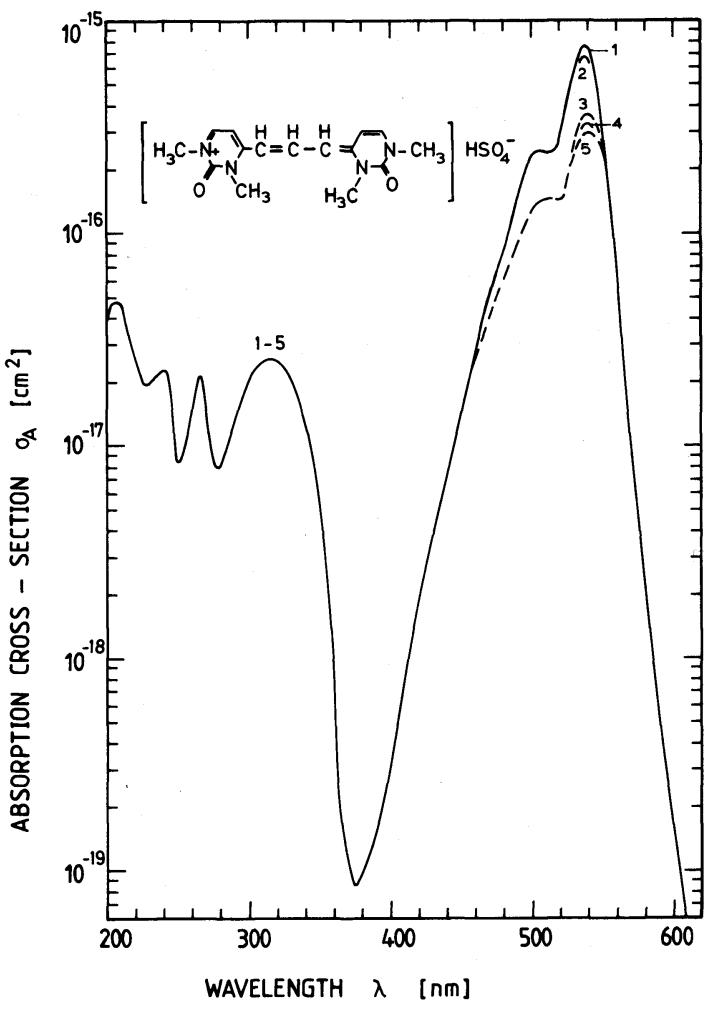


haviour of the dye solution at the phase-matching concentration. In this paper the absorption and emission spectra and the dispersion of the refractive index are measured for several differently concentrated hexafluoroisopropanol solutions. The concentration-dependent changes of the absorption and emission spectra and of the fluorescence quantum efficiencies are analysed. The fluorescence quantum distributions and quantum efficiencies are separated into monomeric and dimeric (closely spaced pair) contributions. The absorption cross sections and the stimulated emission cross sections of the monomers and the closely spaced pairs are resolved. The fluorescence at high concentrations $\left(C>0.014 \mathrm{~mol} / \mathrm{dm}^{3}\right)$ is found to be caused by closely spaced pair emission.

\section{Experiments}

The investigated pyrimidonecarbocyanine dye was supplied by Dr. U. Mayer (BASF). Its structural formula is included in fig. 1. The method of preparation of closely related dyes is described in ref. [5]. The dye is dissolved in 1,1,1,3,3,3-hexafluoro-2-propanol (hexafluoroisopropanol). The dye concentration is varied between $10^{-5}$ and 0.3 $\mathrm{mol} / \mathrm{dm}^{3}$. The experiments are carried out at room temperature.

At low concentrations $\left(C \leqslant 0.01 \mathrm{~mol} / \mathrm{dm}^{3}\right)$ the absorption cross-section spectra, $\sigma_{\mathrm{A}}(\lambda)$, are determined by transmission measurements with a spectrophotometer. The transmission, $T$, is given by $T=\exp (-\alpha l)=\exp \left(-\sigma_{\mathrm{A}} N l\right)$, where $\alpha$ is the absorption coefficient, $\sigma_{\mathrm{A}}$ the absorption cross section, $N$ the number density of dye molecules, and $l$ the sample length. Cell lengths, $l$, down to $10 \mu \mathrm{m}$ have been applied. At high concentrations the absorption cross sections around the $S_{0}-S_{1}$ absorption peak are determined by measuring the reflectivity of parallel-polarized light at the Brewster angle [6]. The analysis of the reflectivity measurements determines both the absorption cross sections, $\sigma_{\mathrm{A}}(\lambda)$, and the refractive indices, $n(\lambda)$.

The fluorescence spectra are measured using front-face illumination in a self-assembled spectrofluorimeter [7]. A spectrally filtered tungsten lamp is used as excitation source. The backward emitted fluorescence light is collected, directed to a spectrometer, and registered with a diode array system. Backward fluorescence detection is necessary for highly concentrated dye solutions in order to minimize fluorescence reabsorption. The determination of the fluorescence quantum distribution, $E(\lambda)\left(\mathrm{nm}^{-1}\right)$, and of the fluorescence quantum efficiency, $q_{\mathrm{F}}=\int_{\mathrm{em}} E(\lambda) \mathrm{d} \lambda$, from the measured fluorescence signal is described in ref. [7]. $E(\lambda)$ is defined as the ratio of the intrinsically generated fluorescence photons per $\mathrm{nm}$ wavelength interval at wavelength $\lambda$ to the absorbed pump light photons.

Additionally the fluorescence anisotropy,

$r_{\mathrm{F}}=\left(I_{\|}-I_{\perp}\right) /\left(I_{\|}+2 I_{\perp}\right)$,

is measured with parallel and perpendicular oriented polarizer sheets in the fluorescence excitation and detection path ( $I_{\|}$: fluorescence signal for parallel polarizers; $I_{\perp}$ fluorescence signal for crossed polarizers). In case of negligible reorientation of the excited molecules the fluorescence anisotropy is $r_{F}=0.4$ for parallel orientation of absorption and emission dipole moment, and $r_{\mathrm{F}}=$ -0.2 for perpendicular orientation between absorption and emission dipole moment $[8,9]$. In case of fast reorientation of the excited molecules within the fluorescence lifetime, $\tau_{\mathrm{F}}$, the fluorescence anisotropy is $r_{\mathrm{F}}=0$.

\section{Results}

The absorption cross-section spectra are shown in fig. 1 for the concentrations of $10^{-4}, 10^{-2}, 0.1$, 0.2 , and $0.3 \mathrm{~mol} / \mathrm{dm}^{3}$. For $C<10^{-4} \mathrm{~mol} / \mathrm{dm}^{3}$ the absorption spectra are concentration independent and are practically identical to the $10^{-4}$ molar spectrum. At high concentrations $\left(C>10^{-3}\right.$ $\mathrm{mol} / \mathrm{dm}^{3}$ ) the $\mathrm{S}_{0}-\mathrm{S}_{1}$ absorption band reduces somewhat while the higher-lying absorption bands $(\lambda<350 \mathrm{~nm})$ remain practically unchanged. The solubility limit of PYC in hexafluoroisopropanol is about $0.4 \mathrm{~mol} / \mathrm{dm}^{3}$ at room temperature.

The reduced $\mathrm{S}_{0}-\mathrm{S}_{1}$ absorption at high concentrations is due to closely spaced pair formation (dye molecules are statistically in close proximity 


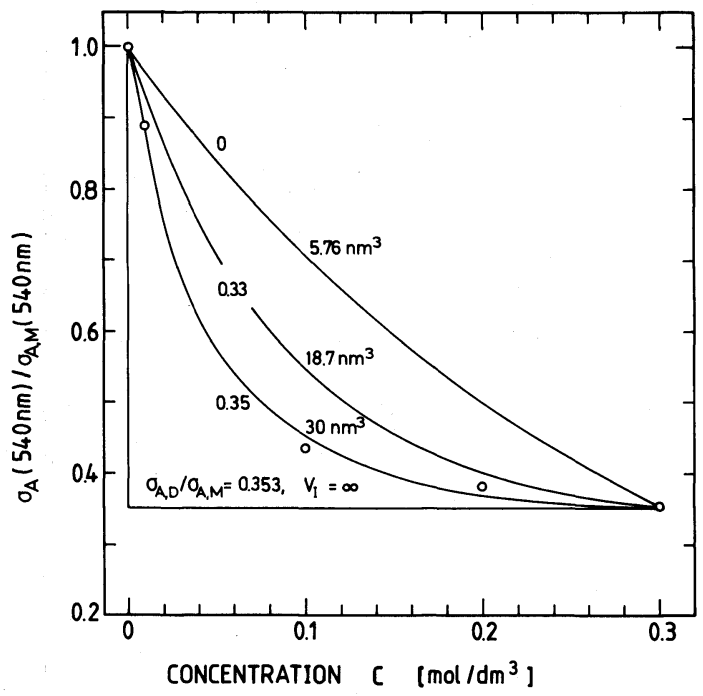

Fig. 2. Absorption cross-section ratio $\sigma_{\mathrm{A}} / \sigma_{\mathrm{A}, \mathrm{M}}$ at $540 \mathrm{~nm}$ versus concentration. The curves are calculated by use of eqs.

(2) and (3) (closely spaced pair model).

and influence one another, e.g., at $C=0.1$ $\mathrm{mol} / \mathrm{dm}^{3}$ the average distance between adjacent molecules is approximately $2.5 \mathrm{~nm}$ ) [10]. At low concentrations only monomers contribute to the absorption. Curve 1 of fig. $1\left(C=10^{-4} \mathrm{~mol} / \mathrm{dm}^{3}\right)$ represents the monomer absorption cross-section spectrum, $\sigma_{\mathrm{A}, \mathrm{M}}(\lambda)$. In fig. 2 the absorption crosssection ratio $\sigma_{\mathrm{A}}(540 \mathrm{~nm}) / \sigma_{\mathrm{A}, \mathrm{M}}(540 \mathrm{~nm})$ is plotted versus concentration. The experimental points are derived from fig. 1 . The curves are calculated by use of the closely spaced pair model (eqs. (9)-(12) of ref. [10]). In this model the absorption is composed of a monomeric (mole fraction $x_{\mathrm{M}}$, cross section $\sigma_{\mathrm{A}, \mathrm{M}}$ ) and a dimeric (mole fraction of molecules in closely spaced pairs: $x_{\mathrm{D}}=1-x_{\mathrm{M}}$, cross section $\sigma_{\mathrm{A}, \mathrm{D}}$ ) contribution. The absorption cross section is given by $\sigma_{\mathrm{A}}=x_{\mathrm{M}} \sigma_{\mathrm{A}, \mathrm{M}}+x_{\mathrm{D}} \sigma_{\mathrm{A}, \mathrm{D}}$. This expression may be rewritten to

$\sigma_{\mathrm{A}} / \sigma_{\mathrm{A}, \mathrm{M}}=1-x_{\mathrm{D}}\left[1-\sigma_{\mathrm{A}, \mathrm{D}} / \sigma_{\mathrm{A}, \mathrm{M}}\right]$.

The fraction, $x_{\mathrm{D}}$, of molecules in closely spaced pairs is [10-12]:

$x_{\mathrm{D}}=1-\exp \left(-V_{\mathrm{I}} N_{\mathrm{A}} C\right)$.

$V_{\mathrm{I}}$ is the interaction volume of a closely spaced pair. $N_{\mathrm{A}}=6.022045 \times 10^{23} \mathrm{~mol}^{-1}$ is the Avogadro number.
The best-fitting curve in fig. 2 gives $\sigma_{\mathrm{A}, \mathrm{D}} / \sigma_{\mathrm{A}, \mathrm{M}}$ $=0.35$ and $V_{\mathrm{I}}=30 \mathrm{~nm}^{3}$. The resulting fraction of molecules in closely spaced pairs, $x_{\mathrm{D}}$, versus concentration is depicted in fig. 3 . Above $0.2 \mathrm{~mol} / \mathrm{dm}^{3}$ nearly all molecules are in closely spaced pairs. The absorption cross-section spectrum of the 0.3 molar solution (curve 5 of fig. 1) is practically identical to the closely spaced pair absorption cross-section spectrum, $\sigma_{\mathrm{A}, \mathrm{D}}(\lambda)$.

The reflectivity of parallel light at the Brewster angle determines the absorption cross section and the refractive index [6]. The refractive index distribution versus wavelength is shown in fig. 4 for the concentrations $C=0.1,0.2$ and $0.3 \mathrm{~mol} / \mathrm{dm}^{3}$. The refractive index dispersion of the solvent hexafluoroisopropanol is included $[3,13]$. The frequency dependence of the refractive index reveals the $\mathrm{S}_{0}-\mathrm{S}_{1}$ absorption peak at $540 \mathrm{~nm}$ and the absorption shoulder at $510 \mathrm{~nm}$ [14].

The fluorescence quantum distributions, $E(\lambda)$, of $10^{-4}, 10^{-2}$, and 0.2 molar dye solutions are shown in fig. 5. The fluorescence quantum distribution at $10^{-5} \mathrm{~mol} / \mathrm{dm}^{3}$ is practically identical to the situation of $10^{-4} \mathrm{~mol} / \mathrm{dm}^{3}$. At $0.1 \mathrm{~mol} / \mathrm{dm}^{3}$

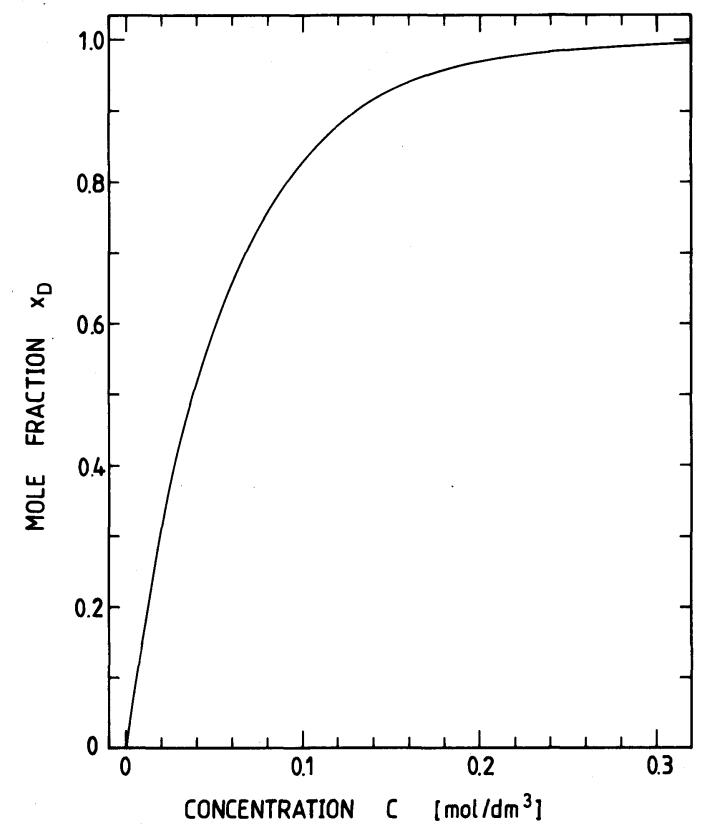

Fig. 3. Mole fraction, $x_{\mathrm{D}}$, of molecules in closely spaced pairs versus concentration. The curve is obtained by use of eq. (3) with interaction volume $V_{\mathrm{I}}=30 \mathrm{~nm}^{3}$. 
and $0.2 \mathrm{~mol} / \mathrm{dm}^{3}$ the fluorescence quantum distributions coincide within experimental error. At high concentrations $E(\lambda)$ is about a factor of 1.9 lower and the vibronic shoulder at $590 \mathrm{~nm}$ is less pronounced than at low concentrations.

The fluorescence quantum efficiency, $q_{\mathrm{F}}=$ $\int_{\mathrm{em}} E(\lambda) \mathrm{d} \lambda$, (integration over $\mathrm{S}_{1}-\mathrm{S}_{0}$ emission band) versus concentration is depicted in fig. 6 (open circles). It reduces from $4 \times 10^{-3}$ at low concentrations $\left(C<10^{-4} \mathrm{~mol} / \mathrm{dm}^{3}\right)$ to about 2.4 $\times 10^{-3}$ at high concentrations $\left(C \geqslant 0.1 \mathrm{~mol} / \mathrm{dm}^{3}\right)$.

The fluorescence quantum efficiency, $q_{\mathrm{F}}(C)$, is composed of the monomer contribution, $q_{\mathrm{M}}(C)$, and the closely spaced pair contribution, $q_{\mathrm{D}}(C)$, according to

$q_{\mathrm{F}}(C)=q_{\mathrm{M}}(C)+q_{\mathrm{D}}(C)$.

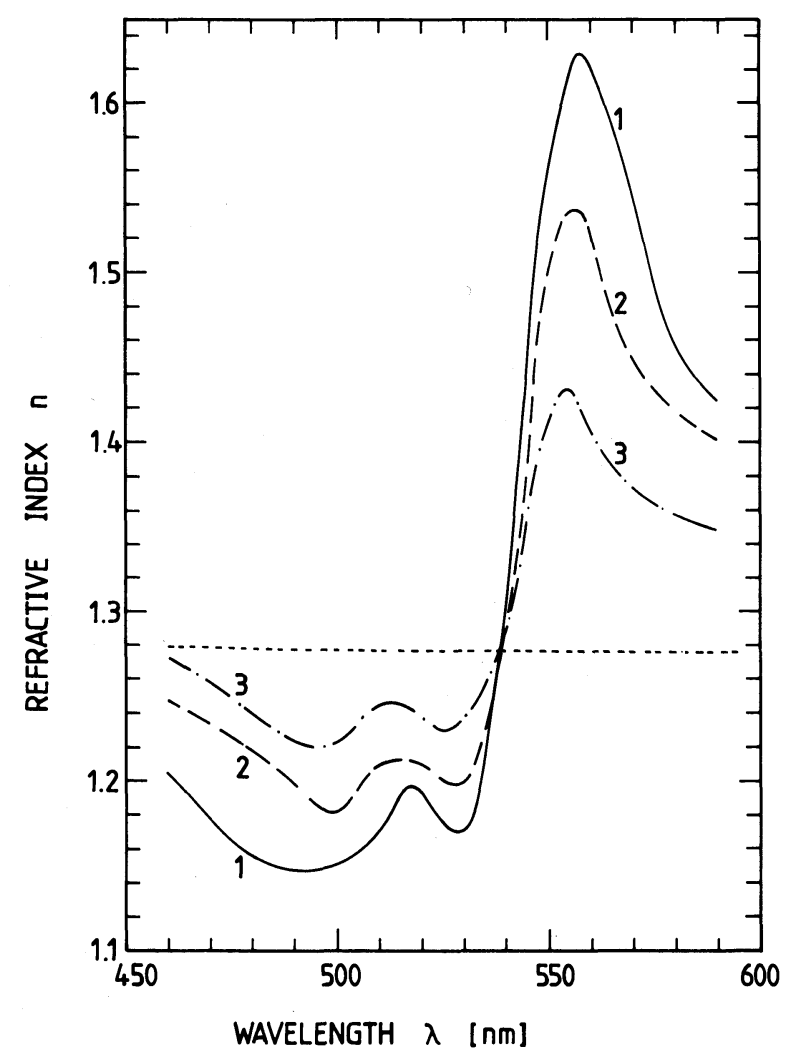

Fig. 4. Dispersion of refractive index of PYC in hexafluoroisopropanol. Curves: (1) concentration $C=0.3 \mathrm{~mol} / \mathrm{dm}^{3},(2)$ $C=0.2 \mathrm{~mol} / \mathrm{dm}^{3}$, and (3) $C=0.1 \mathrm{~mol} / \mathrm{dm}^{3}$. Short dashed curve: solvent hexafluoroisopropanol.
Without energy transfer the quantum efficiencies would be given by

$$
\begin{aligned}
q_{\mathrm{M}}^{\prime}(C) & =\frac{x_{\mathrm{M}} \sigma_{\mathrm{A}, \mathrm{M}}}{x_{\mathrm{M}} \sigma_{\mathrm{A}, \mathrm{M}}+x_{\mathrm{D}} \sigma_{\mathrm{A}, \mathrm{D}}} q_{\mathrm{F}}(0) \\
& =\frac{1-x_{\mathrm{D}}}{1-x_{\mathrm{D}}\left(1-\sigma_{\mathrm{A}, \mathrm{D}} / \sigma_{\mathrm{A}, \mathrm{M}}\right)} q_{\mathrm{F}}(0)
\end{aligned}
$$

and

$$
\begin{aligned}
q_{\mathrm{D}}^{\prime}(C)= & \frac{x_{\mathrm{D}} \sigma_{\mathrm{A}, \mathrm{D}}}{x_{\mathrm{M}} \sigma_{\mathrm{A}, \mathrm{M}}+x_{\mathrm{D}} \sigma_{\mathrm{A}, \mathrm{D}}} q_{\mathrm{F}}\left(x_{\mathrm{D}}=1\right) \\
= & \frac{x_{\mathrm{D}}}{\sigma_{\mathrm{A}, \mathrm{M}} / \sigma_{\mathrm{A}, \mathrm{D}}-x_{\mathrm{D}}\left(\sigma_{\mathrm{A}, \mathrm{M}} / \sigma_{\mathrm{A}, \mathrm{D}}-1\right)} \\
& \times q_{\mathrm{F}}\left(x_{\mathrm{D}}=1\right) .
\end{aligned}
$$

The pre-factors in eqs. (5) and (6) give the ratio of the number of excited monomers and closely

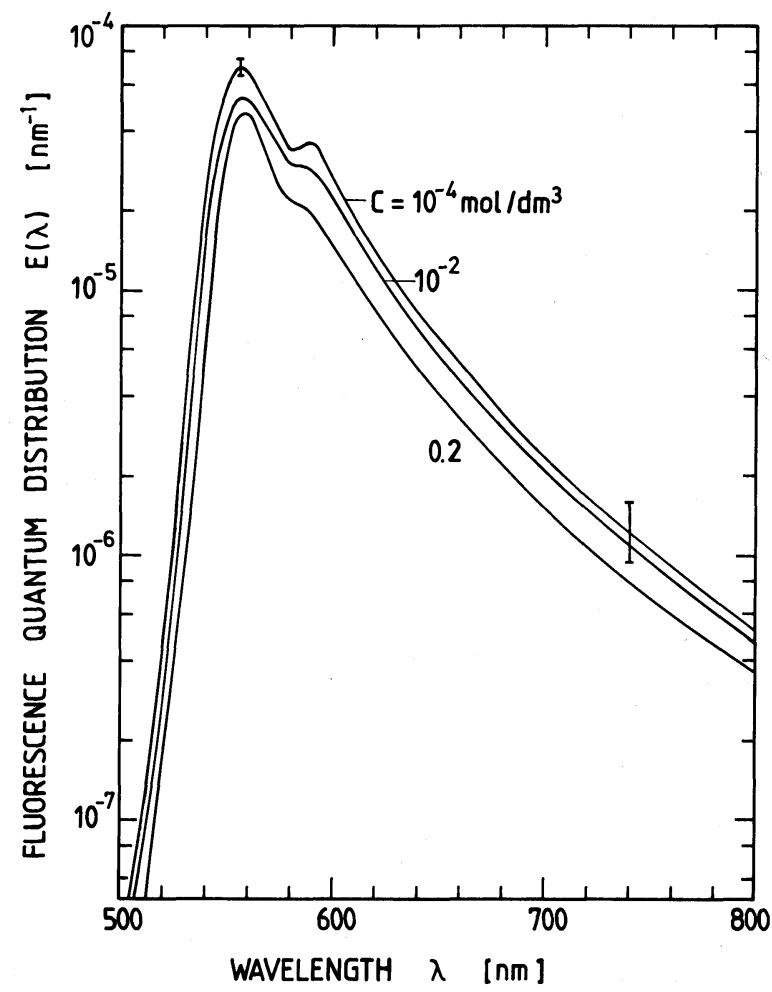

Fig. 5. Fluorescence quantum distributions, $E(\lambda)$, for three different concentrations of PYC in hexafluoroisopropanol. The upper curve $\left(C=10^{-4} \mathrm{~mol} / \mathrm{dm}^{3}\right)$ represents the monomer fluorescence quantum distribution, $E_{M}(\lambda)$; the lower curve $\left(C=0.2 \mathrm{~mol} / \mathrm{dm}^{3}\right)$ represents the closely spaced pair distribution, $E_{\mathrm{D}}(\lambda)$. 


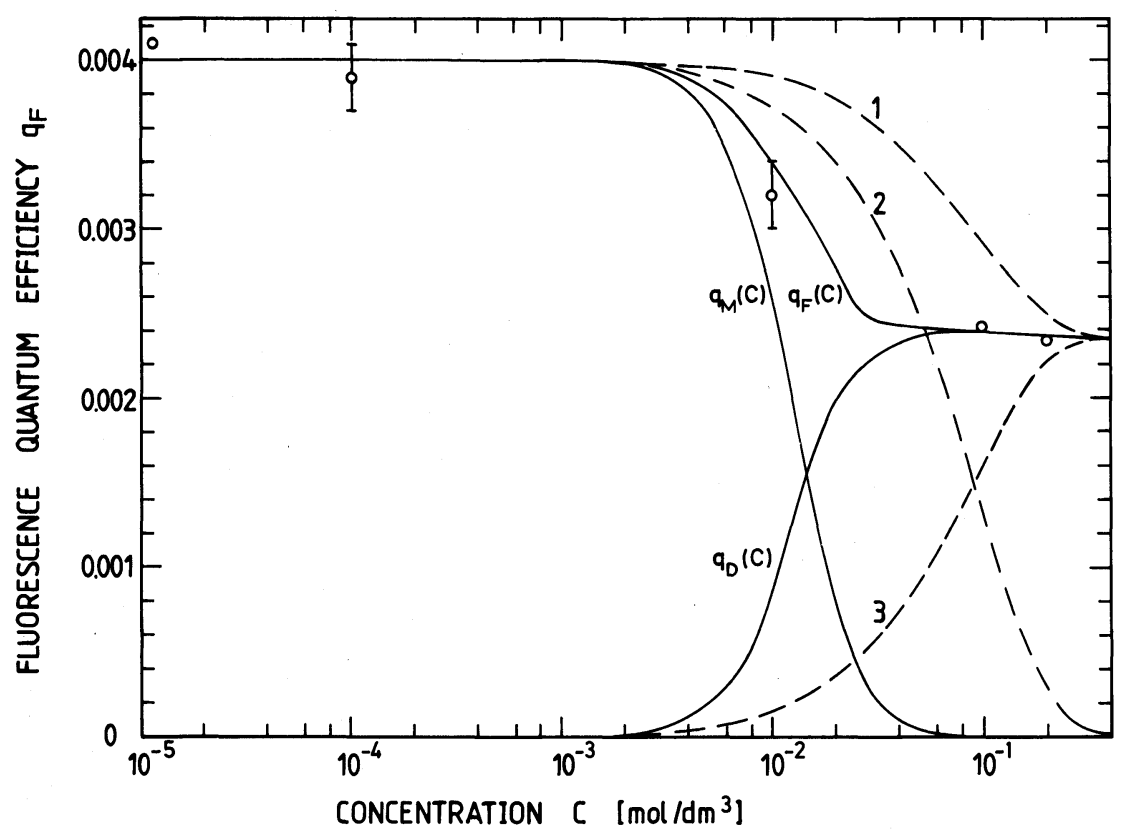

Fig. 6. Fluorescence quantum efficiency versus concentration of PYC in hexafluoroisopropanol. Open circles are experimental points. The curves are calculated. Dashed curves, without energy transfer. (1) $q_{\mathrm{F}}^{\prime}(C)$; (2) $q_{\mathrm{M}}^{\prime}(C)$; (3) $q_{\mathrm{D}}^{\prime}(C)$. Solid curves include Förster-type energy transfer.

spaced pairs, respectively, to the total number of excited molecules. $\sigma_{\mathrm{A}, \mathrm{M}}$ and $\sigma_{\mathrm{A}, \mathrm{D}}$ are the monomer and closely spaced pair absorption cross sections at the frequency of the fluorescence excitation source $(\approx 530 \mathrm{~nm}$ in our experiments). Curves 1 , 2 , and 3 of fig. 6 represent $q_{\mathrm{F}}^{\prime}(C)=q_{\mathrm{M}}^{\prime}(C)+$ $q_{\mathrm{D}}^{\prime}(C), q_{\mathrm{M}}^{\prime}(C)$, and $q_{\mathrm{D}}^{\prime}(C)$, respectively. The deviation of $q_{\mathrm{F}}^{\prime}(C)$ from the experimental points is due to the neglection of energy transfer.

Inclusion of the Förster-type energy transfer from monomers to closely spaced pairs [11,12] leads to a monomer relaxation rate, $k_{\mathrm{M}}(C)$, of

$$
\begin{aligned}
k_{\mathrm{M}}(C) & =k_{\mathrm{R}, 0}+k_{\mathrm{NR}, 0}+k_{E}(C) \\
& =k_{\mathrm{R}, 0}\left[1+\frac{k_{\mathrm{NR}, 0}}{k_{\mathrm{R}, 0}}+x_{\mathrm{D}}\left(\frac{C}{C_{0}}\right)^{2}\right] .
\end{aligned}
$$

$k_{\mathrm{R}, 0}$ and $k_{\mathrm{NR}, 0}$ are the radiative and radiationless relaxation rates, respectively. $k_{E}(C)=k_{\mathrm{R}, 0} x_{\mathrm{D}^{-}}$ $\times\left(C / C_{0}\right)^{2}$ is the Förster-type energy transfer rate. $C_{0}$ is the critical transfer concentration. The monomer fluorescence quantum efficiency is $q_{\mathrm{M}}(C)=\left(x_{\mathrm{M}} \sigma_{\mathrm{A}, \mathrm{M}} /\left[x_{\mathrm{M}} \sigma_{\mathrm{A}, \mathrm{M}}+x_{\mathrm{D}} \sigma_{\mathrm{A}, \mathrm{D}}\right]\right) k_{\mathrm{R}, 0} / k_{\mathrm{M}}(C)$.
Using $\quad q_{\mathrm{F}}(0)=k_{\mathrm{R}, 0} / k_{\mathrm{M}}(0)=\left(1+k_{\mathrm{NR}, 0} / k_{\mathrm{R}, 0}\right)^{-1}$ the result is

$$
\begin{aligned}
q_{\mathrm{M}}(C)= & \frac{1-x_{\mathrm{D}}}{1-x_{\mathrm{D}}\left(1-\sigma_{\mathrm{A}, \mathrm{D}} / \sigma_{\mathrm{A}, \mathrm{M}}\right)} \\
& \times \frac{q_{\mathrm{F}}(0)}{1+x_{\mathrm{D}}\left(C / C_{0}\right)^{2} q_{\mathrm{F}}(0)} .
\end{aligned}
$$

The closely spaced pair fluorescence quantum efficiency is

$$
\begin{aligned}
q_{\mathrm{D}}(C)= & q_{\mathrm{D}}^{\prime}(C)+\left[q_{\mathrm{M}}^{\prime}(C)-q_{\mathrm{M}}(C)\right] \\
& \times q_{\mathrm{F}}\left(x_{\mathrm{D}}=1\right) / q_{\mathrm{F}}(0) .
\end{aligned}
$$

The total fluorescence quantum efficiency, $q_{\mathrm{F}}(C)$, is given by eq. (4). The solid curves in fig. 6 represent $q_{\mathrm{F}}(C), q_{\mathrm{M}}(C)$, and $q_{\mathrm{D}}(C)$. The fitting parameter $C_{0}=3.5 \times 10^{-4} \mathrm{~mol} / \mathrm{dm}^{3}$ is used in the calculations. The corresponding Förster energy transfer radius is $R_{0}=\left[\frac{4}{3} \pi N_{\mathrm{A}} C_{0}\right]^{-1 / 3} \approx 10 \mathrm{~nm}$.

Energy back-transfer from closely spaced pairs to monomers is not included in eqs. (7)-(9) (for inclusion see ref. [12]). An energy back-transfer is 


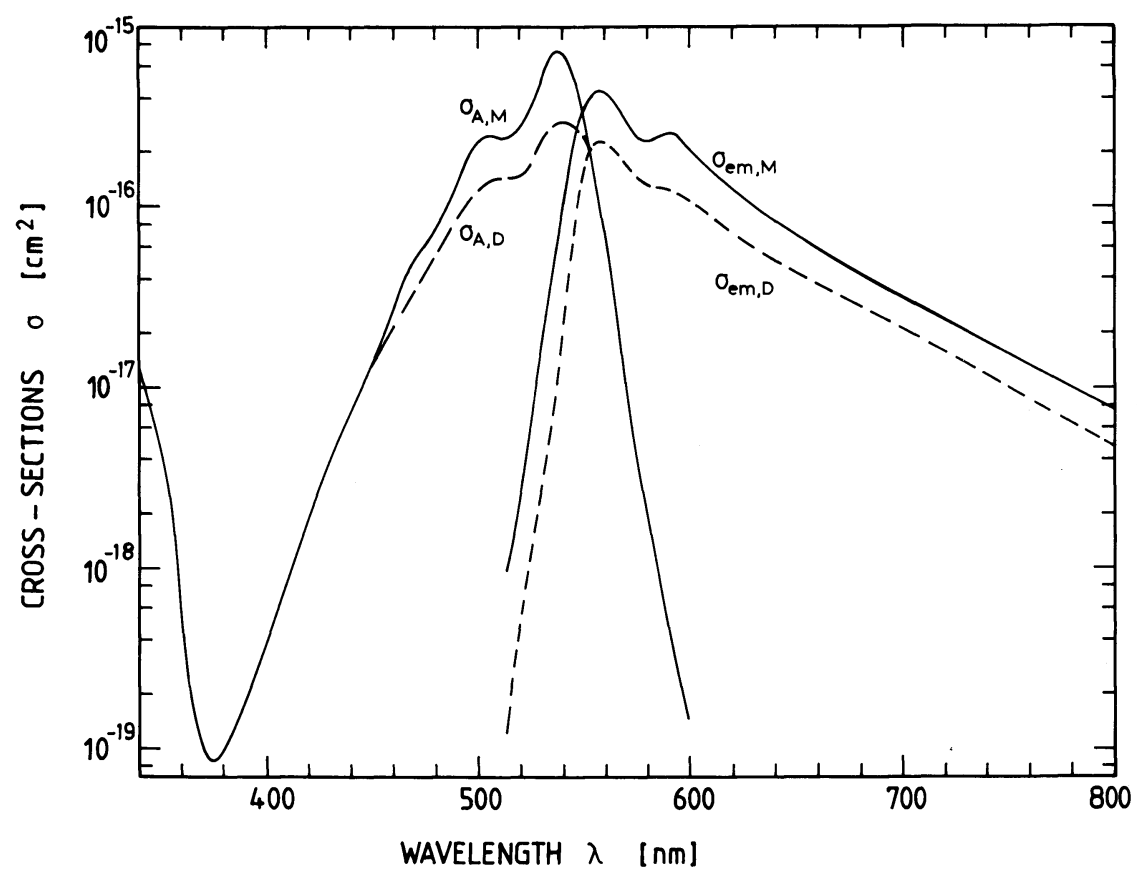

Fig. 7. Monomer and closely spaced pair absorption and stimulated emission cross sections of PYC in hexafluoroisopropanol.

expected because the closely spaced pair spectra are only slightly shifted to longer wavelength (fig. 7) giving still a good overlap between the closely spaced pair emission spectrum and the monomer absorption spectrum. The energy back-transfer does not strongly influence $q_{\mathrm{F}}(C)$ since $q_{\mathrm{F}}(0)$ and $q_{\mathrm{F}}\left(x_{\mathrm{D}}=1\right)$ are not very different $\left(q_{\mathrm{M}}=q_{\mathrm{F}}(0)\right.$ $\left.=0.004, q_{\mathrm{D}}=q_{\mathrm{F}}\left(x_{\mathrm{D}}=1\right)=0.0024\right)$.

The fluorescence quantum distribution, $E(\lambda)$, is composed of a monomeric, $E_{M}(\lambda)$, and a dimeric, $E_{\mathrm{D}}(\lambda)$, contribution, i.e.

$$
\begin{aligned}
E(\lambda, C)= & E_{\mathrm{M}}(\lambda, C)+E_{\mathrm{D}}(\lambda, C) \\
= & {\left[q_{\mathrm{M}}(C) / q_{\mathrm{F}}(0)\right] E(\lambda, 0) } \\
& +E_{\mathrm{D}}(\lambda, C) .
\end{aligned}
$$

At $C=0.1$ and $0.2 \mathrm{~mol} / \mathrm{dm}^{3}$ the measured fluorescence quantum distribution is equal to the closely spaced pair fluorescence quantum distribution, $E_{\mathrm{D}}(\lambda)=E_{\mathrm{F}}\left(\lambda, x_{\mathrm{D}}=1\right)$.

The fluorescence anisotropy, $r_{\mathrm{F}}$, was determined for the dye concentrations $C=10^{-4}$ $\mathrm{mol} / \mathrm{dm}^{3}$ and $C=0.08 \mathrm{~mol} / \mathrm{dm}^{3}$. The obtained values are $r_{\mathrm{F}}\left(10^{-4} \mathrm{~mol} / \mathrm{dm}^{3}\right)=0.4 \pm 0.05$ and
$r_{\mathrm{F}}\left(0.08 \mathrm{~mol} / \mathrm{dm}^{3}\right)=0.0 \pm 0.05$. At low concentrations (result of $C=10^{-4} \mathrm{~mol} / \mathrm{dm}^{3}$ ) the fluorescence anisotropy is maximal. The $S_{0}-S_{1}$ absorption and $S_{1}-S_{0}$ emission transition dipole moments are parallel to each other and reorientation of the transition dipoles is negligible within the fluorescence lifetime $\tau_{\mathrm{F}}\left(\tau_{\mathrm{F}} \approx 13 \mathrm{ps}\right.$, see below; the reorientation time, $\tau_{\text {or }}$, is expected to be $\tau_{\text {or }} \geq$ 100 ps [15], viscosity of hexafluoroisopropanol at $25^{\circ} \mathrm{C}$ is $1.62 \mathrm{cP}$ [13]). At high concentrations (result of $C=0.08 \mathrm{~mol} / \mathrm{dm}^{3}$ ) the fluorescence signal is completely depolarized $\left(r_{\mathrm{F}}=0\right)$. The fast Förster-type energy transfer between excited and unexcited molecules $\left(k_{E}>\tau_{\mathrm{F}}^{-1}\right)$ at high concentration $[11,12,7]$ depolarizes the fluorescence emission ( $r_{\mathrm{F}} \rightarrow 0$, independent of solvent viscosity).

The radiative lifetimes of the monomers, $\tau_{\text {rad,M }}$ $=k_{\mathrm{R}, 0}^{-1}$, and of the closely spaced pairs, $\tau_{\mathrm{rad}, \mathrm{D}}$, are given by the Strickler-Berg formula $[16,17](i=\mathrm{M}$ or D):

$$
\frac{1}{\tau_{\mathrm{rad}, i}}=\frac{8 \pi n_{\mathrm{F}}^{3} c_{0}}{n_{\mathrm{A}}} \frac{\int_{\mathrm{em}} E_{i}(\lambda) \lambda \mathrm{d} \lambda}{\int_{\mathrm{em}} E_{i}(\lambda) \lambda^{4} \mathrm{~d} \lambda} \int_{\mathrm{abs}} \frac{\sigma_{\mathrm{A}, i}(\lambda)}{\lambda} \mathrm{d} \lambda .
$$


$c_{0}$ is the vacuum light velocity. $n_{\mathrm{A}}$ and $n_{\mathrm{F}}$ are the average refractive indices of the solution in the $\mathrm{S}_{0}-\mathrm{S}_{1}$ absorption and emission band, respectively. The results of PYC in hexafluoroisopropanol are $\tau_{\text {rad,M }}=3.3 \mathrm{~ns}$ and $\tau_{\text {rad,D }}=4.1 \mathrm{~ns}$. The StricklerBerg formula gives reliable radiative lifetimes if the absorption cross-section spectrum and the fluorescence quantum distribution spectrum exhibit a mirror image shape as is the case for PYC in hexafluoroisopropanol (figs. 1 and 5).

In case of single exponential decay of the $S_{1}$ level population of the monomers $\left(x_{D}=0\right)$ and the dimers $\left(x_{\mathrm{D}}=1\right)$, the fluorescence lifetimes are related to the radiative lifetimes by $\tau_{\mathrm{F}, i}=q_{\mathrm{F}, i} \tau_{\mathrm{rad}, i}$. Under this assumption the fluorescence lifetimes of the monomers and the closely spaced pairs are $\tau_{\mathrm{F}, \mathrm{M}}=q_{\mathrm{M}} \tau_{\mathrm{rad}, \mathrm{M}}=13 \pm 2 \mathrm{ps}$ and $\tau_{\mathrm{F}, \mathrm{D}}=q_{\mathrm{D}} \tau_{\mathrm{rad}, \mathrm{D}}=$ $10 \pm 2$ ps.

The stimulated emission cross-section spectra, $\sigma_{\mathrm{em}, \mathrm{M}}(\lambda)$ and $\sigma_{\mathrm{em}, \mathrm{D}}(\lambda)$, of the monomers $(i=\mathrm{M})$ and the closely spaced pairs $(i=\mathrm{D})$ are given by [18]:

$\sigma_{\mathrm{em}, i}(\lambda)=\frac{\lambda^{4} E_{i}(\lambda)}{8 \pi n_{\mathrm{F}}^{2} c_{0} q_{\mathrm{F}, i} \tau_{\mathrm{rad}, i}}$.

The $\mathrm{S}_{0}-\mathrm{S}_{1}$ absorption and emission cross-section spectra of the monomers and closely spaced pairs are plotted in fig. 7. The total integrated cross sections of the closely spaced pairs are about a factor of 1.9 lower than the values of the monomers $\left[\int_{\mathrm{abs}} \sigma_{\mathrm{A}, \mathrm{M}}(\tilde{\nu}) \mathrm{d} \tilde{\nu}=1 \times 10^{-12} \mathrm{~cm} ; \int_{\mathrm{abs}} \sigma_{\mathrm{A}, \mathrm{D}}\right.$ $(\tilde{\nu}) \mathrm{d} \tilde{\nu}=5.5 \times 10^{-13} \mathrm{~cm} ; \int_{\mathrm{em}} \sigma_{\mathrm{em}, \mathrm{M}}(\tilde{\nu}) \mathrm{d} \tilde{\nu}=8.6 \times$ $10^{-13} \mathrm{~cm} ; \int_{\mathrm{em}} \sigma_{\mathrm{em}, \mathrm{D}}(\tilde{\nu}) \mathrm{d} \tilde{\nu}=4.5 \times 10^{-13} \mathrm{~cm}, \tilde{\nu}=$ $\lambda^{-1}$ ]. The $S_{0}-S_{1}$ transition dipole moment of the closely spaced pairs seems to be reduced compared to the monomers. Similar reductions of the absorption strengths of dimers were reported in refs. [19-24]. The frequency positions of the absorption peak and the vibronic shoulder of the closely spaced pairs are about $2 \mathrm{~nm}$ shifted to longer wavelengths. The ratio of the absorption cross sections of the vibronic shoulder to the absorption peak is increased in the closely spaced pairs $\left[\sigma_{\mathrm{A}, \mathrm{D}}(510 \mathrm{~nm}) / \sigma_{\mathrm{A}, \mathrm{D}}(542 \mathrm{~nm})=0.48 ; \sigma_{\mathrm{A}, \mathrm{M}}\right.$ $\left.(508 \mathrm{~nm}) / \sigma_{\mathrm{A}, \mathrm{M}}(540 \mathrm{~nm})=0.28\right]$. The enlarged $\mathrm{ab}-$ sorption of the vibronic shoulder compared to the absorption peak indicates a slightly larger
Franck-Condon shift (difference in equilibrium position between $S_{1}$ state and $S_{0}$ state) of the closely spaced pairs compared to the monomers [7,10,25-27].

\section{Conclusions}

The linear spectroscopic behaviour of a pyrimidonecarbocyanine dye in hexafluoroisopropanol is investigated. From the measured absorption and emission spectra, the monomer and closely spaced pair absorption and emission cross sections are resolved, the monomer and closely spaced pair fluorescence quantum distributions and quantum efficiencies are determined. In highly diluted hexafluoroisopropanol solutions the radiationless relaxation of the $S_{1}$ state is dominant leading to a short fluorescence lifetime of $\tau_{\mathrm{F}} \approx 13$ ps. For concentrations above $0.1 \mathrm{~mol} / \mathrm{dm}^{3}$ the fluorescence is identified as closely spaced pair emission with a fluorescence lifetime of $\tau_{\mathrm{F}} \approx 10$ ps. Because of the strong $S_{0}-S_{1}$ absorption and the short $S_{1}$-state lifetime, PYC in hexafluoroisopropanol is a potential saturable absorber in the wavelength region from 480 to $610 \mathrm{~nm}[28,29]$ (see also ref. [30]). The application of PYC for efficient phase-matched third-harmonic generation with picosecond $\mathrm{Nd}$ lasers was already mentioned in section 1 [1].

\section{Acknowledgement}

The authors are very indebted to Dr. U. Mayer of BASF Ludwigshafen for providing the dye and for helpful discussion. They are grateful to Dr. H.J. Sasse (BASF) for valuable information. They thank the Deutsche Forschungsgemeinschaft and the Fonds der chemischen Industrie for financial support and the Rechenzentrum of the University of Regensburg for disposal of computer time.

\section{References}

[1] W. Leupacher, A. Penzkofer, B. Runde and K.H. Drexhage, Appl. Phys. B., to be published. 
[2] P.P. Bey, I.E. Giuliani and H. Rabin, Phys. Rev. Letters 19 (1967) 819.

[3] J.C. Diels and F.P. Schäfer, Appl. Phys. 5 (1974) 197.

[4] L.I. Al'perovich, T.B. Bavaev and V.V. Shabalov, J. Appl. Spectry. USSR 26 (1977) 196.

[5] H. Baumann, G. Hansen, H.-R. Müller and M. Seefelder, Ann. Chem. 717 (1968) 124.

[6] Y. Lu and A. Penzkofer, Appl. Opt. 25 (1986) 221.

[7] A. Penzkofer and W. Leupacher, J. Luminescence (1987), to be published.

[8] F. Dörr, Angew. Chem. 78 (1966) 457.

[9] E.D. Cehelnik, K.D. Mielenz and R.A. Velapoldi, J. Res. Natl. Bur. Std. 79 A (1975) 1.

[10] Y. Lu and A. Penzkofer, Chem. Phys. 107 (1986) 175.

[11] Th. Förster, Fluoreszenz organischer Verbindungen (Vandenhoeck and Ruprecht, Göttingen, 1951).

[12] A. Penzkofer and Y. Lu, Chem. Phys. 103 (1986) 399.

[13] Technical report DP-4A of DuPont on hexafluoroisopropanol (1968).

[14] W. Leupacher and A. Penzkofer, Appl. Opt. 23 (1984) 1554.

[15] H.E. Lessing and A. von Jena, in: Laser handbook, Vol. 3, ed. M.L. Stitch (North-Holland, Amsterdam, 1979) ch. B6.

[16] S.J. Strickler and R.A. Berg, J. Chem. Phys. 37 (1962) 814.
[17] J.B. Birks and D.J. Dyson, Proc. Roy. Soc. A 275 (1963) 135.

[18] O.G. Peterson, J.B. Webb, W.C. McColgin and J.H. Eberly, J. Appl. Phys. 42 (1971) 1917.

[19] J. Tinoco, J. Am. Chem. Soc. 82 (1960) 4785.

[20] W. Rhodes, J. Am. Chem. Soc. 83 (1961) 3609.

[21] M.E. Lamm and D.M. Neville, J. Phys. Chem. 69 (1965) 3872.

[22] A.R. Monahan and D.F. Blossey, J. Phys. Chem. 74 (1970) 4014.

[23] M.G. Reva, L.V. Levshin and B.D. Ryzhikov, J. Appl. Spectry. USSR 33 (1979) 1091.

[24] V.I. Zemskii, I.K. Meshkovskii and I.A. Sokolov, Opt. Spectry. (USSR) 59 (1985) 197.

[25] V. Zanker, M. Held and H. Rammensee, Z. Naturforsch. 14b (1959) 789.

[26] E.A. Chandross and J. Ferguson, J. Chem. Phys. 45 (1966) 4532.

[27] M. Pope and C.E. Swenberg, Electrical processes in organic crystals (Clarendon Press, Oxford, 1982).

[28] W. Blau, W. Dankesreiter and A. Penzkofer, Chem. Phys. 85 (1984) 473.

[29] A. Penzkofer and P. Sperber, Chem. Phys. 88 (1984) 309.

[30] A. Penzkofer, Appl. Phys. B 40 (1986) 85. 